\title{
Funciones sobre los enteros y saltamontes
}

\author{
Adán Medrano Martín del Campo
}

\begin{abstract}
Resumen
En este artículo se presentan dos problemas que involucran funciones definidas sobre los enteros positivos. Cada problema puede ser interpretado como una sucesión de saltos de un saltamontes.
\end{abstract}

Palabras y frases clave: Función, Función discreta, Enteros, Residuos, Sucesión, Descenso, Inducción.

\section{Functions over the integers and grasshoppers}

\begin{abstract}
In this paper two problems about functions defined over the positive integers are presented. Each problem can be interpreted as a sequence of jumps of a grasshopper.
\end{abstract}

Key words and phrases: Function, Discrete function, Integers, Residues, Sequence, Descend, Induction.

\section{Introducción}

Las ecuaciones funcionales y problemas de funciones en general son una tendencia actual en las olimpiadas de matemáticas. Una manifestación de esto es la cantidad de problemas de este estilo que han aparecido en las recientes IMO (International Mathematical Olympiad). Por esta razón es importante pasar una cantidad considerable de tiempo jugando con las ideas que conllevan las soluciones de estos problemas, pues estas son muy variadas.

En este artículo hablaremos de un tipo particular de funciones: aquellas definidas sobre los enteros positivos. Estas funciones son discretas, pues su dominio lo es, y por lo tanto las técnicas de resolución que uno emplea difieren de las técnicas usadas en otros dominios, como los son los números reales o racionales. En particular, los problemas de los cuales hablaremos tienen cierta relación con ideas proviniendo de teoría de números.

El primer problema del cual hablaremos apareció en la Olimpiada Mexicana de Matemáticas (OMM) de 2019 como problema 5. Este no habla propiamente sobre 
una función, si no de un saltamontes. Sin embargo, la idea detrás de la solución puede ser formalizada de manera simple construyendo una función apropiada. El segundo problema cae en la categoría opuesta: se habla de una función, pero le daremos una interpretación usando un saltamontes. Este problema apareció en el examen de selección de equipo (TST) de Rumania para la IMO de 2019.

\section{Problemas}

Procedamos a los enunciados de los dos problemas de los que hablaremos.

Problema 1 (OMM 2019, P5). Sean $a>b$ dos números enteros positivos, primos relativos entre sí. En un camino recto, en el cual está marcado cada centímetro $n$, para todo entero $n$, un saltamontes hará algunos saltos comenzando en la marca de $0 \mathrm{~cm}$ y siguiendo las siguientes reglas:

- Cuando cierto minuto sea múltiplo de $a$ y no múltiplo de $b$, saltará $a$ centímetros hacia adelante.

- Cuando cierto minuto sea múltiplo de $b$ y no múltiplo de $a$, saltará $b$ centímetros hacia atrás.

- Cuando cierto minuto sea múltiplo de a y múltiplo de $b$, saltará $a-b$ centímetros hacia adelante.

- Cuando un minuto no es múltiplo de $a$ ni de $b$, el saltamontes no se mueve del lugar en el que está.

Determina todas las marcas a las que puede llegar el saltamontes.

Sin hablar sobre la solución a dicho problema, el enunciado puede traducirse al siguiente problema equivalente:

Problema 1'. Sean $a>b$ enteros primos positivos primos relativos entre sí y sea $f: \mathbb{N} \rightarrow \mathbb{Z}$ la función dada por

$$
f(n)=a\left\lfloor\frac{n}{a}\right\rfloor-b\left\lfloor\frac{n}{b}\right\rfloor .
$$

Determina la imagen de $f$.

Uno puede jugar un poco con la función definida arriba, y llegar a la respuesta usando propiedades de dicha función. El objetivo de mostrarles este enunciado equivalente, es 
que muchas veces ciertos problemas que hablan de ciertos procesos pueden describirse (y resolverse) en términos de funciones construidas apropiadamente.

El segundo problema que resolveremos cae en la categoría opuesta, pues es un problema sobre una función, al cual se le puede dar una interpretación de los saltos de un saltamontes.

Problema 2 (Romania TST 2019, P3): Sean $a<b<c$ enteros positivos y sea $f: \mathbb{N} \rightarrow \mathbb{N}$ una función dada por

$$
f(n)= \begin{cases}n-a & n>c \\ f(f(n+b)) & n \leq c\end{cases}
$$

Determina la cantidad de enteros positivos $n$ tales que $f(n)=n$.

En este momento, no parece que este problema tenga que ver con un saltamontes. Sin embargo, miremos la siguiente interpretación:

Problema 2': Sean $a<b<c$ enteros positivos. Un saltamontes se encuentra sobre un entero $n>0$ en la recta real positiva, donde hay pasto en los enteros positivos menores o iguales que $c$, y lava en los enteros mayores a $c$. Inicialmente, el saltamontes tiene una vida, y mientras el saltamontes tenga al menos una vida, se dispondrá a saltar de la siguiente manera:

- Si el saltamontes se encuentra en el pasto, el saltamontes gana una vida y salta $b$ enteros hacia adelante.

- Si el saltamontes se encuentra en la lava, el saltamontes pierde una vida y salta $a$ enteros hacia atrás.

Cuando el saltamontes tiene 0 vidas, este muere y deja de moverse. Determina todas las posiciones iniciales del saltamontes tal que el saltamontes morirá en su posición inicial.

En la figura 2 podemos observar una ilustración de la situación anterior, cortesía de Leonardo Ignacio Martínez Sandoval.

\section{Soluciones}

Ahora procedamos a resolver el primer problema 


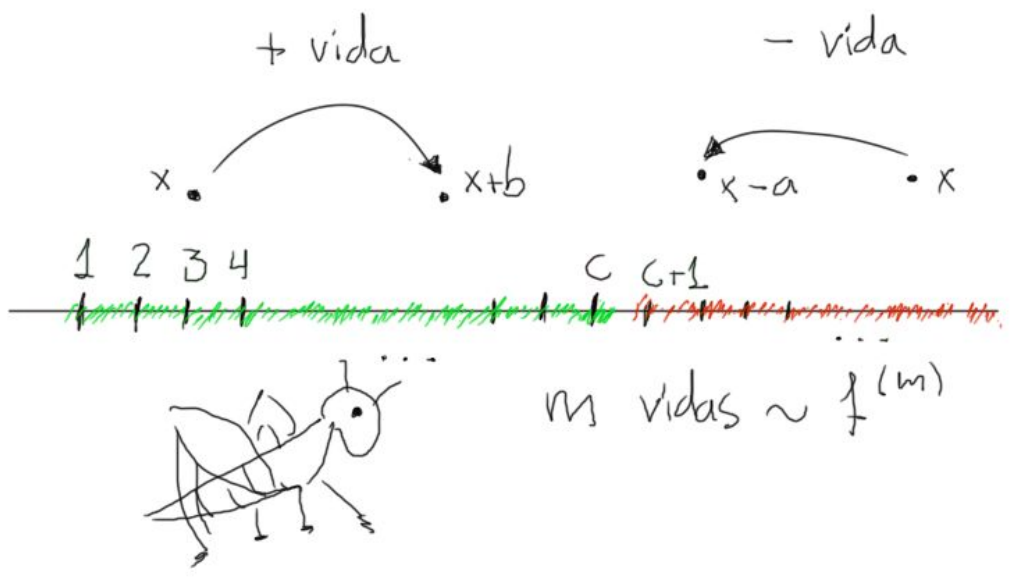

Figura 1: Ilustración sel segundo problema interpretado con saltamontes

Solución al problema 1. Notemos que al dividir $n$ entre $a$ y entre $b$, obtenemos

$$
n=a\left\lfloor\frac{n}{a}\right\rfloor+r_{a}
$$

$\mathrm{y}$

$$
n=b\left\lfloor\frac{n}{b}\right\rfloor+r_{b}
$$

donde

$$
0 \leq r_{a} \leq a-1
$$

$\mathrm{y}$

$$
0 \leq r_{b} \leq b-1
$$

son precisamente los residuos que resultan de la división. Notemos entonces que

$$
\begin{aligned}
f(n) & =a\left\lfloor\frac{n}{a}\right\rfloor-b\left\lfloor\frac{n}{b}\right\rfloor \\
& =\left(n-b\left\lfloor\frac{n}{b}\right\rfloor\right)-\left(n-a\left\lfloor\frac{n}{a}\right\rfloor\right) \\
& =r_{b}-r_{a}
\end{aligned}
$$

por lo que $f(n)$ simplemente depende de la diferencia entre $r_{b}$ y $r_{a}$. Por el Teorema Chino del Residuo, o simplemente mirando exclusivamente a los múltiplos de $a$ y de $b$ entre 1 y $a b$, aparecen como diferencia todos los posibles enteros en el intervalo

$$
[-a+1, b-1]
$$


lo cual compone la imagen de $f$, que es lo que buscábamos.

Mirar los residuos fue clave en este problema. En particular, no lo usamos en nuestra solución, pero $f$ resulta ser una función periódica, con periodo $a b$. Esto es gracias a que $a$ y $b$ son primos relativos, y por lo tanto cada pareja de residuos $r_{a}, r_{b}$ se repiten exactamente cada $a b$ enteros.

La periodicidad será una propiedad clave en la solución del segundo problema. Comenzamos mostrando una exploración del problema.

Solución al problema 2. Los puntos $n$ tales que $f(n)=n$ son llamados puntos fijos. En la formulación como problema de saltamontes, corresponden a que el saltamontes muera justo donde empezó: muera es que ya no haya $f$, empieza con una vida, osea una $f$. Comenzaremos con este pequeño lema.

Lema 1. Si $n>c$, entonces $n$ no es un punto fijo de $f$.

Demostración. Notemos que si $n>c$, entonces

$$
f(n)=n-a \neq n
$$

Esto nos dice que los puntos fijos son menores o iguales que $c$. Ahora, notemos que (recordemos que $a<b<c$ )

$$
\begin{aligned}
f(c) & =f(f(c+b)) \\
& =f(c+b-a)=c+b-2 a
\end{aligned}
$$

y esto nos lleva a considerar que números cercanos a $c$, dentro de un intervalo de tamaño $b-a$, tendrán un valor similar. En efecto, si $0 \leq r<b-a$ entonces

$$
\begin{aligned}
f(c-r) & =f(f(c-r+b)) \\
& =f(c-r+b-a) \\
& =c-r+b-2 a .
\end{aligned}
$$

Ahora, veamos que restando $b-a$ a $c$, perdemos este patrón, pues

$$
\begin{aligned}
f(c-b+a) & =f(f(c+a)) \\
& =f(c) \\
& =c+b-2 a
\end{aligned}
$$


¡Hemos regresado a un valor ya conocido! Esto nos lleva a la hipótesis de que $f$ es periódica con periodo $b-a$ en el intervalo $[1, c]$. La manera de enunciar formalmente las observaciones anteriores es, por ejemplo, via el siguiente lema:

Lema 2. Sea $n=c-r-k(b-a)$ un entero positivo menor o igual que $c$ donde $k \geq 0$ y $0 \leq r<b-a$. Entonces

$$
f(n)=c-r+b-2 a .
$$

Demostración. Procederemos por descenso en los enteros positivos. Construiremos una secuencia de valores iguales, con distinta cantidad de $f^{\prime}$ 's compuestas, de la siguiente manera: comenzamos con

$$
z_{0}=n=c-r-k(b-a)
$$

y definimos

$$
z_{i+1}= \begin{cases}z_{i}-a & z_{i}>c \\ z_{i}+b & z_{i} \leq c\end{cases}
$$

para todo $i \geq 0$. Además, escribiremos

$$
z_{i}=c-r-y_{i} b+x_{i} a
$$

donde $x_{0}=y_{0}=k, \mathrm{y}$ ambas secuencias $\left\{x_{i}\right\}$ y $\left\{y_{i}\right\}$ decrecen, definiendo

$$
x_{i+1}= \begin{cases}x_{i}-1 & z_{i}>c \\ x_{i} & z_{i} \leq c\end{cases}
$$

y

$$
y_{i+1}= \begin{cases}y_{i} & z_{i}>c \\ y_{i}-1 & z_{i} \leq c\end{cases}
$$

Habiendo definido esto, tenemos que

$$
f(n)=f^{\left(1+x_{i}-y_{i}\right)}\left(z_{i}\right)
$$

para todo $i \geq 0$. Observemos que si $y_{i}=-1$ entonces $z_{i}=c-r+b+x_{i} a>c$ si se cumple que $x_{i} \geq-1$. Más aún, observemos el siguiente lema:

Lema 3. Para todo $i \geq 0$, tenemos que $y_{i} \geq 0$ implica que $y_{i+1} \leq x_{i+1}$. 
Demostración. Procedemos por inducción. Para $i=0$ esto es claro, pues

$$
y_{1}=k-1<k=x_{1} .
$$

Ahora, supongamos que $x_{i} \geq y_{i} \geq 0$. Si $x_{i}>y_{i}$ entonces

$$
x_{i+1} \geq x_{i}-1 \geq y_{i} \geq y_{i+1} .
$$

Si $x_{i}=y_{i}$ entonces tenemos que

$$
z_{i}=c-r-y_{i}(b-a) \leq c
$$

por lo que $z_{i+1}=z_{i}+b$ y esto implica que

$$
x_{i+1}=x_{i}>y_{i}-1=y_{i+1} .
$$

Hemos probado pues que las secuencias $\left\{x_{i}\right\}$ y $\left\{y_{i}\right\}$ decrecen, y mientras $y_{i} \geq 0$, tendremos que $x_{i+1} \geq y_{i+1}$. La clave ahora es notar la existencia de la menor $m$ tal que $y_{m}=-1$, donde es claro que $y_{m-1}=0$. Si $m=1$ entonces $y_{0}=x_{0}=k=0$, y ya hemos cubierto ese caso arriba, así que asumiremos que $m>1$. Tenemos que $y_{m-2} \geq 0$ por lo que, por el lema 3 ,

$$
x_{m-1} \geq y_{m-1}=0
$$

y como $y_{m}=y_{m-1}-1$ entonces $x_{m}=x_{m-1} \geq 0$. Esto implica que

$$
\begin{aligned}
z_{m} & =c-r+b+x_{m} a \\
& \geq c-r+b \\
& >c
\end{aligned}
$$

por lo que para todo $j>m$ se tiene que $x_{j+1}=x_{j}-1$

$$
z_{m+x_{m}+1}=c-r+b-a
$$

y tenemos que $y_{m+x_{m}+1}=x_{m+x_{m}+1}=-1$, lo que muestra que

$$
\begin{aligned}
f(n) & =f\left(z_{m+x_{m}+1}\right) \\
& =f(c-r+b-a) \\
& =c-r+b-2 a .
\end{aligned}
$$


ISSN: 2711-1792 (En línea) • Espacio Matemático Vol. 1 No. 2 (2020), pp. 104111

Hemos mostrado la periodicidad de $f$. Falta únicamente usar esto para hacer una conclusión sobre los puntos fijos. Notemos que los únicos valores de $f$ en el dominio $[1, c]$ son $c-r+b-2 a$ para $0 \leq r<b-a$, así que solo estos valores pueden ser puntos fijos de $f$. De hecho, cada uno de esos valores es un punto fijo si y solo si podemos encontrar una $k \geq 0$ tal que

$$
c-r-k(b-a)=c-r+b-2 a
$$

lo cual sucede si y sólo si $(k+1)(b-a)=a$, o bien justo cuando $b-a \mid a$, por lo que si $b-a$ divide a $a$, todos nuestros $b-a$ valores son puntos fijos, y si $b-a$ no divide a $a$, ningún valor es un punto fijo. Hemos concluido entonces.

\section{Agradecimientos}

Esta exposición surgió, en cierto modo, a causa del COVID-19 y la cuarentena que esta provocó. Junto con Leonardo Ignacio Martínez Sandoval, David Guadalupe Torres Flores, y Pablo Meré Hidalgo, administro el grupo de facebook InsOMMnia, el cual sirve de plataforma para discutir y realizar actividades olímpicamente productivas relacionadas a la Olimpiada Mexicana de Matemáticas. A modo de amenizar la cuarentena, hice un video en vivo explicando la solución a estos problemas, pues me parecieron agradables por varias razones:

- El primer problema apareció en la $33^{\circ}$ Olimpiada Mexicana de Matemáticas, celebrada en Ciudad de México en Noviembre de 2019.

- El segundo problema fue parte del selectivo del equipo rumano para la 59 IMO, la cual fue celebrada en Bath, Reino Unido en Julio de 2019.

Tras esta exposición, Leonardo, uno de los administradores antes mencionado, me invitó a hacer una entrada en su blog, El blog de Leo. Posteriormente, Leonardo también me sugirió exponer las notas que escribí en su blog para esta revista. Extiendo mi agradecimiento a todos los mencionados.

\section{Referencias}

[1] Medrano, A., Un Problema de Saltamontes en Cuarentena, Blog Post (2020, Marzo 31), extraido de http://blog.nekomath.com/un-problema-desaltamontes-en-cuarentena/. 
ISSN: 2711-1792 (En línea) • Espacio Matemático Vol. 1 No. 2 (2020), pp. 104,111

[2] Olimpiada Mexicana de Matemáticas 2019, Problema 5 (2019, Noviembre 21) Another grasshopper problem, extraido de https://artofproblemsolving.com/community/c6h1951252p13469477

[3] Romania Team Selection Test 2019, Day 1, Problem 3 (2019, Junio 19) number of fixed points of a function, extraido de https://artofproblemsolving.com/community/c6h1858830p12566844

Adán Medrano Martín del Campo (amedrano@math.uchicago.edu)

The University of Chicago 